\title{
DECELERATION OF A FAST ION IN AN ACCRETION COLUMN
}

J.G. Kirk.

Max-Planck-Institut für Physik und Astrophysik, Föhringer Ring 6, 8 Mïnchen 40, FRG.

Calculations of the rate of energy deposition by a fast ion falling through the plasma above a magnetised neutron star are important in the physics of X-ray pulsars, since they play a decisive role in the question of the formation of a stand-off shock front. Treatments of spherically symmetric accretion (Alme and Wilson, 1973) used the formula for deceleration by Coulomb collisions in the absence of a magnetic field. This approach requires modification when a magnetised neutron star is considered. Basko and Syunyaev (1975) and Pavlov and Yakovlev (1976) calculated the relevant cross-sections, and estimated that the stopping length of an ion is increased by an order of magnitude over the zero-field case. However, this conclusion no longer holds when the collective effects in the plasma are included. The most import of these is the scattering of the test ion off the ion-sound waves. That such a scattering can occur is seen only when full account is taken of the orbit of the ion in the magnetic field. Even a modest level of ion-sound turbulence suffices to deflect the ion, and severely reduce the stopping length. Computer calculations of the energy and momentum deposition rates including this new effect are in progress, and it is hoped that the results will indicate whether or not a non-radiative shock can occur.

\section{References}

Alme, M.I. and Wilson, J.R. 1973 Astrophys. J. 186,1015

Basko, M.M. and Syunyaev, R.A. 1975 Sov. Phys. JETP $\underline{41}, 52$

Pavlov, G.G. and Yakovlev, D.G. 1976 Sov. Phys. JETP $\underline{43}, 489$ 